
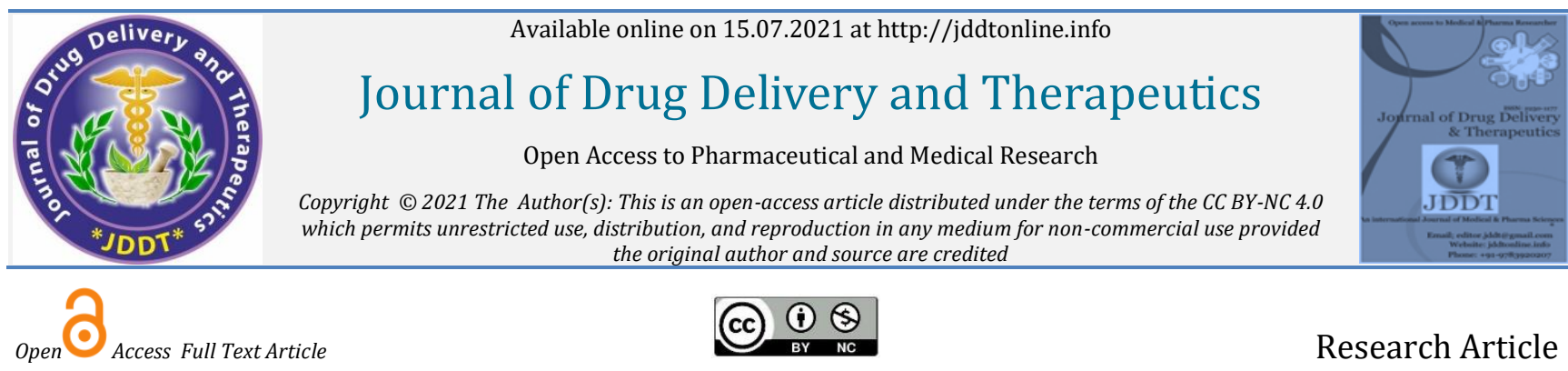

Research Article

\title{
Profile of Bioactive Phyto-compounds in Essential Oil of Cymbopogon martinii from Palani Hills, Western Ghats, INDIA
}

\author{
C. Soorya ${ }^{1}$, S. Balamurugan ${ }^{1}$, Afroze Naveed Basha², C. Kandeepan², S. Ramya1 ${ }^{1}$ R. Jayakumararaj1* \\ ${ }^{1}$ Department of Botany, Government Arts College, Melur- 625106, Madurai District, Tamil Nadu, India
}

${ }^{2}$ PG and Research Department of Zoology, Arulmigu Palaniandavar College of Arts \& Culture, Palani - 624601, Dindigul District, TamilNadu, India

Article Info:
Received 19 May 2021
Acview Completed 24 June 2021
Available online 15 July 2021

\section{Abstract}

Worldwide interest in use of plants based natural products (PBNPs) has been growing, and its beneficial effects being rediscovered for the development of novel drugs. Literature survey on indigenous traditional knowledge bestows ethnopharmacological potentials of PBNPs that has inspired current research in drug design and discovery; PBNPs provide baseline for the development of novel drug leads against various pharmacological targets. Studies indicate that Cymbopogon martini Essential Oil (CMEO) exhibit wide range of biological activities such as hepatoprotective, antifungal, insecticide, antioxidant and antibacterial. Pharmacological properties in Palmarosa Essential Oil (PEO) may be due to the presence of compounds like 4-Decen-6-yne, (Z), 2-Ethylimino-4-methyl-pent-3enenitrile, Dihydrocarvyl acetate, 2-Methylbenzaldehyde, Geranyl butyrate, 1,5,9,9Tetramethyl-1,4,7-cycloundecatriene. However, its application is limited because of the odor, color and taste. In the present study, GCMS based profile of bioactive phytocompounds in essential oil of Cymbopogon martinii along with its physiochemical, biological, molecular, pharmacological and drugable properties has been envisaged.

Keywords: Cymbopogon martinii Essential Oil (CMEO); Pharmacological Activity; ADMET Properties; Bioactive Compounds; Plant Based Natural Products (PBNPs);

\section{INTRODUCTION}

Genus Cymbopogon is widely distributed in the tropical and subtropical regions of Africa, Asia and America. The genus Cymbopogon comprises of more than 144 species, and is well known for its high content of essential oils ${ }^{1,2}$. Studies have led to the isolation of alkaloids, volatile and non-volatile terpenoids, flavonoids, carotenoids and tannins from every part of Cymbopogon species. Cymbopogon martinii (lemongrasses) is native to India and Indochina, but widely cultivated in many places for its aromatic essential oil. Known as Palmarosa, the plant has other names: Indian geranium, ginger grass, rosha, and rosha grass. Besides, therapeutic application, it is commonly used as a condiment and food preservative. PEO contains bioactive molecules, phyto-compounds, endowed with pharmacological activities $^{3}$. PEO contains geraniol, used as scent and in a number of traditional medicinal. PEO is of commercial importance, being extensively used in perfumes, soaps, cosmetics, toiletry and tobacco products 4 . PEO has effective insect repellent property when applied to stored grain and beans ${ }^{5}$, antihelmintic against nematodes ${ }^{5}$, antifungal ${ }^{6,7}$ and mosquito repellent ${ }^{8}$ activity. CMEO is used in aromatherapy due to its antimicrobial properties. It is used in Ayurvedic medicine to treat skin problems and relieve nerve pain. Immunomodulatory action of CMEO was evaluated towards production of pro- and anti-inflammatory cytokines (TNF- $\alpha$ and IL-10) by human monocytes in vitro ${ }^{9}$.

Essential Oils (EOs) a major group of Phytogenic Bio-Active Compounds (PBAC) have been used for variety of purposes. Due to their physiochemical properties and bioactive nature, EOs has been used in aromatherapy, as flavor and fragrances in cosmetics, foods, and more recently as pharmaceuticals, natural preservatives, additives, and biopesticides ${ }^{10,11}$. EOs are concentrated form of liquid mixtures of volatile compounds of plant origin with unique structural chemistry including terpenoid and non-terpenoid hydrocarbons and their oxygenated derivatives, with natural color, odor and flavor, or "essence" of their source - volatile/ odoriferous oil. EOs are extracted from various plant parts such as leaves, fruit, bark, root, wood, heartwood, gum, balsam, berries, seeds, flowers, twigs, and buds ${ }^{12}$.

Role of EOs in drug development has been well documented since antiquity nevertheless, they are directly used as therapeutic agents due to fact that they have proven record in traditional indigenous systems of medicine such as Ayurveda, Siddha, Unani and Homeopathy and in modern medicine, EOs contain bioactive compounds of GRAS nature. Furthermore, concern about the negative effect of synthetic chemicals as food additives warrants "GO" products with no or lesser side effects. Therefore, growing interest in natural 
extracts as alternatives for synthetic additives is attributed to (a) their synergy with other preservation methods (b) generally regarded as safe, and (c) PBNPs are endowed with antioxidant, antidiabetic, antimutagenic, antitoxigenic and antibacterial properties. Apart from effective antioxidants of CMEO viz., cyclic diterpene diphenols, carnosolic acid and carnosol CMEO contains carnosic acid, epirosmanol rosmanol, methylcarnosate and isorosmanol ${ }^{13}$ however, needs scientific validation ${ }^{14}$.

Cymbopogon martinii (Palmarosa) has been traced for its origin from the Mediterranean region. It is an aromatic plant, a unique spice commercially available for use as an antioxidant. CMEO extracts have been used in the treatment of diseases, due to its phytotherapeutic potential ${ }^{14}$. On the other hand, it is used in food preservation, PEO could even decrease the use of synthetic antioxidants in foods. EFSA (European Food Safety Authority) recently, reviewed the safety of CMEO extracts and concluded that there are highintake estimates ranging from 0.09 (elderly) to 0.81 (children) $\mathrm{mg} / \mathrm{kg}$ per day.

\section{Cymbopogon martini (PALMAROSA)}

Botanical Description: Perennial from a short woody rootstock. Culms tufted, up to $3 \mathrm{~m}$ tall, lower nodes often swollen, mealy. Leaf sheaths glabrous; leaf blades lanceolate, usually glaucous below, dark green above, up to $50 \times 2-3 \mathrm{~cm}$, glabrous, base cordate, often amplexicaul, apex filiform; ligule 2-4 $\mathrm{mm}$. Spathate panicle narrow, dense, erect, 20-30 $\mathrm{cm}$; spatheoles green becoming reddish, $2-4 \mathrm{~cm}$; racemes $1.5-2 \mathrm{~cm}$; rachis internodes and pedicels ciliate on margins, back sometimes pubescent; pedicel of homogamous pair swollen, barrel-shaped, shiny, fused to internode at base. Sessile spikelet oblong, 3.5-4.5 mm; lower glume flat, deeply grooved below middle, keels winged above middle, vein less or 2-veined between keels; upper lemma 2-lobed; awn 1.4$1.8 \mathrm{~cm}$. Pedicelled spikelet $3.5-4 \mathrm{~mm}$. Fl. and fr. Jul-Oct. This grass is native to India, but is cultivated elsewhere in tropical region of the world for its essential oils.

In traditional medicine both the plant and its oils are used to treat rheumatism, hair loss, arthritis, lumbago and spasms. The essential oil is a strong fungicide. In laboratory tests it was more effective than several synthetic fungicides against pathogenic fungi and yeasts, including Aspergillus spp., Candida albicans, Monilia sitophila and Trichophyton tonsurae ${ }^{14,15}$. In Ayurvedic medicine - Charak gave the decoction of whole plant in the treatment of abdominal disorders, the liver disorders, jaundice, fever and disorders of the spleen. In Sushruta, decoction of whole plant is prescribed in inflammation of throat, chest pain, indigestion, bronchitis, cough and asthma.

\section{MATERIALS AND METHODS}

\section{Collection, Preparation and Extraction of Oil from the sample}

The leaf samples were collected from wild in the Perumalmalai Region (Perumalmalai is a hillock in the Palani Hills, Dindigul District, TamilNadu) Western Ghats, INDIA during December 2020. The leaf sample were well preserved, taken to laboratory, identified by using flora ${ }^{16,17}$ shade dried and processed as per the protocol for preparation of sample according to the methods previously described by Eleyinmi ${ }^{18}$, however, with modifications in the temperature and duration of processing of the sample. As much as $100 \mathrm{~g}$ leaf was weighed and dried in an oven at $60^{\circ} \mathrm{C}$. Dried sample was ground into powder using ThomasWilley milling machine and sieved on a wire mesh screen $(3$ $\times 3 \mathrm{~mm}^{2}$ ). Sample was stored at $4^{\circ} \mathrm{C}$ in air-tight container with screw caps. Sample was prepared according to the methods previously described by Rašković et al.13. $25 \mathrm{~g}$ of sample was suspended in $250 \mathrm{~mL}$ of distilled water in stoppered flasks and allowed to stand for $24 \mathrm{~h}$, filtered with Whatman No 24 filter paper, concentrated in a rotary evaporator for $12 \mathrm{~h}$ at $50^{\circ} \mathrm{C}$ and dried in vacuum desiccator. Yield was calculated to be $6.06 \% \mathrm{w} / \mathrm{w}$. Extract was suspended in ethyl acetate and subjected to GC-MS analysis.

\section{GC-MS Analysis}

Cymbopogon martini (Palmarosa) Essential Oil was extracted, from the leaf samples collected from the Perumalmalai Region, Palani, Dindigul District, Tamil Nadu, India. Phyto-components were identified using GC-MS detection system as described previously, however with modification, whereby portion of the extract was analysed directly by headspace sampling. GC-MS analysis was accomplished using an Agilent 7890A GC system set up with 5975C VL MSD (Agilent Technologies, CA, and USA). Capillary column used was DB-5MS $(30 \mathrm{~m} \times 0.25 \mathrm{~mm}$, film thickness of $0.25 \mu \mathrm{m}$; J\&W Scientific, CA, USA). Temperature program was set as follows: initial temperature $50^{\circ} \mathrm{C}$ held for $1 \mathrm{~min}, 5^{\circ} \mathrm{C}$ per min to $100^{\circ} \mathrm{C}, 9^{\circ} \mathrm{C}$ per min to $200^{\circ} \mathrm{C}$ held for $7.89 \mathrm{~min}$, and the total run time was $30 \mathrm{~min}$. The flow rate of helium as a carrier gas was $0.811851 \mathrm{~mL} / \mathrm{min}$. MS system was performed in electron ionization (EI) mode with Selected Ion Monitoring (SIM). The ion source temperature and quadruple temperature were set at $230^{\circ} \mathrm{C}$ and $150^{\circ} \mathrm{C}$, respectively. Identification of phyto-components was performed by comparison of their retention times and mass with those of authentic standards spectra using computer searches in NIST 08.L and Wiley 7n.l libraries.

\section{ADMET prediction}

Selected phytocompounds were subjected to ADMET prediction using QikProp (version 4.3, Suite 2015-1; Schrödinger, LLC: New York, NY) and toxicity prediction using TOPKAT (Accelrys, Inc., USA). QikProp develops and employs QSAR/QSPR models using partial least squares, principal component analysis and multiple linear regression to predict physic-chemically significant descriptors ${ }^{19}$.

\section{RESULTS AND DISCUSSION}

\section{GCMS analysis}

The chemical composition of EOs depends on plant genetics, growth conditions, development stage at harvest, and processes of extracting active compounds. Different parts of the plant (bark, leaf, fruit and seed) have been extensively investigated for their bioactive phytochemical constituents in various plants ${ }^{20}$. GC-MS analysis revealed that the extract of Cymbopogon martini contained different volatile oils (Jummes et al., 2020). 4-Decen-6-yne, ( $\mathrm{Z})-\left(\mathrm{C}_{10} \mathrm{H}_{16}\right), 3.568$ min, 10 hits; 2-Ethylimino-4-methyl-pent-3-enenitrile $\left(\mathrm{C}_{8} \mathrm{H}_{12} \mathrm{~N}_{2}\right), 3.913$ min, 10 hits; Cyanogen bromide $(\mathrm{CBrN})$, 4.024, 1 hits; Cyclohexanol, 2-methyl-5-(1-methylethenyl)-, (1.alphA.,2.betA. ,5.alphA. ) - $\left(\mathrm{C}_{10} \mathrm{H}_{18} \mathrm{O}\right), 4.503$ min, 10 hits; Cyclohexa-1,3-diene, 5,6-diethyl- $\left(\mathrm{C}_{10} \mathrm{H}_{16}\right), 4.915 \mathrm{~min}, 10$ hits; Benzaldehyde, 2-methyl- $\left(\mathrm{C}_{8} \mathrm{H}_{8} \mathrm{O}\right), 8.154 \mathrm{~min}, 10$ hits; Pyrazine $\left(\mathrm{C}_{4} \mathrm{H}_{4} \mathrm{~N}_{2}\right), 9.32,5$ hits; 2-Norbornaneacetic acid $\left(\mathrm{C}_{9} \mathrm{H}_{14} \mathrm{O}_{2}\right), \quad 9.378,8$ hits; cis-syn-trans-Tricyclo[7.3.0.0 $(2,6)]$ dodec-7-ene $\left(\mathrm{C}_{12} \mathrm{H}_{18}\right), 9.509 \mathrm{~min}, 10$ hits; 1,2,4Metheno-1H-indene, octahydro-1,7a-dimethyl -5-(1methylethyl)-, [1S (1.alphA. ,2.alphA. ,3A. betA. ,4.alphA. ,5.alphA. ,7A. be tA. ,8S*)]- ( $\left.\mathrm{C}_{15} \mathrm{H}_{24}\right), 9.913 \mathrm{~min}, 10$ hits; 1,4,7, Cycloun-decatriene, 1,5,9,9-tetramethyl-Z,Z,Z- $\quad\left(\mathrm{C}_{15} \mathrm{H}_{24}\right)$, 10.343 min, 10 hits; Naphthalene, decahydro-4a-methyl-1methylene- 7-(1-methylethylidene)-, (4aR-trans)- $\left(\mathrm{C}_{15} \mathrm{H}_{24}\right)$, $10.738 \mathrm{~min}, 10$ hits; Butanoic acid, 3,7-dimethyl-2,6- 
octadienyl ester,(E)- $\left(\mathrm{C}_{14} \mathrm{H}_{24} \mathrm{O}_{2}\right), 11.772 \mathrm{~min}, 10$ hits; Nerolidol $2\left(\mathrm{C}_{15} \mathrm{H}_{26} \mathrm{O}\right), 11.948$ min, 10 hits; Caryophyllene oxide $\left(\mathrm{C}_{15} \mathrm{H}_{24} \mathrm{O}\right), 12.525 \mathrm{~min}, 10$ hits; 2-Azidomethyl-1,3,3trimethyl-cyclohexene $\left(\mathrm{C}_{10} \mathrm{H}_{17} \mathrm{~N}_{3}\right), 15.152 \mathrm{~min}, 10$ hits; Hexanoic acid, 3,7-dimethyl-2,6-octadienyl ester, (E)$\left(\mathrm{C}_{16} \mathrm{H}_{28} \mathrm{O}_{2}\right), 15.423 \mathrm{~min}, 10$ hits; Hexanoic acid, 3,7-dimethyl2,6-octadienyl ester, (E)- $\left(\mathrm{C}_{16} \mathrm{H}_{28} \mathrm{O}_{2}\right), 15.701$ min, 10 hits; Farnesol, acetate $\left(\mathrm{C}_{17} \mathrm{H}_{28} \mathrm{O}_{2}\right), 17.258 \mathrm{~min}, 10$ hits; 2,6Octadien-1-ol, 3,7-dimethyl-, propanoate, (Z)- $\left(\mathrm{C}_{13} \mathrm{H}_{22} \mathrm{O}_{2}\right)$, 20.158, 10 hits respectively (Table 1 ).

Biological activities of these secondary metabolites of Cymbopogon martini (Palmarosa) have been reported for its antitumor, antioxidant, anti-infectious, anti-inflammatory, and analgesic activities and effects on the central nervous system, endocrine system, disorders such ascardiac remodeling after myocardial infarction, body weight changes, dyslipidemia, cerebral ischemia, hepatonephrotoxicity, stress, and anxiety. Anti-inflammatory activity of CMEO has been attributed to the presence and synergistic activity of carnosol and carnosic, rosmarinic, ursolic, oleanolic, and micromeric acids (A). Specifically, anti-inflammatory activity has been attributed to synergic effects of ursolic and micromeric acids present in CMEO ${ }^{21}$. These natural drugs can be proposed for preclinical and clinical studies in different diseases and pathological conditions.

2D, 3D structures of bioactive compounds in C. martini essential oil is given in Table 1. Molecular and biological properties (CID, MF, miLogP, TPSA, N atoms, MW (g/mol), Non, n OHNH, N violations, N rotb, volume, GPCR ligand, Ion channel modulator, Kinase inhibitor, Nuclear receptor ligand, Protease inhibitor, Enzyme inhibitor) of the bioactive compounds is provided in Table 2. Summary of toxic (mutagenic, toxicology, irritant, reproductive properties) risk assessment towards drugability/ drug score in Table 3 indicates that the compounds were neither mutagenic nor toxic to biological system. All the bioactive compounds studied were drugable candidates and had good score for Druggability Properties - Lipinski's rule of 5 violations, Veber rule, Egan rule, Oral PhysChem score, GSK's 4/400 score, Pfizer's 3/75 score, QEDw score, Solubility, Solubility Index (Table 4).

Similarly, ADMET properties of key molecules in CMEO (Caryophyllene oxide and Geranyl butyrate) towards Human Intestinal Absorption, Blood Brain Barrier, Caco-2 permeable, P-glycoprotein substrate, P-glycoprotein inhibitor I, P-glycoprotein inhibitor II, CYP450 2C9 substrate, CYP450 2D6 substrate, CYP450 3A4 substrate, CYP450 1A2 inhibitor, CYP450 2C9 inhibitor, CYP450 2D6 inhibitor, CYP450 2C19 inhibitor, CYP450 3A4 inhibitor, CYP450 inhibitory promiscuity, Ames test, Carcinogenicity, Biodegradation, Rat acute toxicity, LD50 mol/kg, hERG inhibition (predictor I), hERG inhibition (predictor II) (Table 5) indicate that these molecules can be used for drug formulations.

Table 1: GC-MS profile of compounds $s$ in C. martini essential oil

\begin{tabular}{|c|c|c|c|}
\hline RT & Name of the Compound & $\begin{array}{l}\text { Molecular } \\
\text { Formula }\end{array}$ & $\begin{array}{l}\text { Hits } \\
\text { (DB) }\end{array}$ \\
\hline 3.568 & 4-Decen-6-yne, (Z)- & $\mathrm{C}_{10} \mathrm{H}_{16}$ & 10 \\
\hline 3.913 & 2-Ethylimino-4-methyl-pent-3-enenitrile & $\mathrm{C}_{8} \mathrm{H}_{12} \mathrm{~N}_{2}$ & 10 \\
\hline 4.024 & Cyanogen bromide & $\mathrm{CBrN}$ & 1 \\
\hline 4.503 & Cyclohexanol, 2-methyl-5-(1-methylethenyl)(1.alpha.,2.beta.,5.alpha.)- & $\mathrm{C}_{10} \mathrm{H}_{18} \mathrm{O}$ & 10 \\
\hline 4.915 & Cyclohexa-1,3-diene, 5,6-diethyl- & $\mathrm{C}_{10} \mathrm{H}_{16}$ & 10 \\
\hline 8.154 & Benzaldehyde, 2-methyl- & $\mathrm{C}_{8} \mathrm{H}_{8} \mathrm{O}$ & 10 \\
\hline 9.32 & Pyrazine & $\mathrm{C}_{4} \mathrm{H}_{4} \mathrm{~N}_{2}$ & 5 \\
\hline 9.378 & 2-Norbornaneacetic acid & $\mathrm{C}_{9} \mathrm{H}_{14} \mathrm{O}_{2}$ & 8 \\
\hline 9.509 & cis-syn-trans-Tricyclo[7.3.0.0(2,6)]dodec-7-ene & $\mathrm{C}_{12} \mathrm{H}_{18}$ & 10 \\
\hline 9.913 & $\begin{array}{l}\text { 1,2,4-Metheno-1H-indene, octahydro-1,7a- } \\
\text { dimethyl-5-(1-methylethyl)-, [1S- (1.alpha.,2.alpha.,3a.beta.,4.alpha.,5.alpha.,7a.be ta.,8S*)]- }\end{array}$ & $\mathrm{C}_{15} \mathrm{H}_{24}$ & 10 \\
\hline 10.343 & 1,4,7,-Cycloundecatriene, 1,5,9,9-tetramethyl- & $\mathrm{C}_{15} \mathrm{H}_{24}$ & 10 \\
\hline 10.738 & $\begin{array}{l}\text { Naphthalene, decahydro-4a-methyl-1-methylene- } \\
\text { 7-(1-methylethylidene)-, (4aR-trans)- }\end{array}$ & $\mathrm{C}_{15} \mathrm{H}_{24}$ & 10 \\
\hline 11.772 & Butanoic acid, 3,7-dimethyl-2,6-octadienyl ester,(E)- & $\mathrm{C}_{14} \mathrm{H}_{24} \mathrm{O}_{2}$ & 10 \\
\hline 11.948 & Nerolidol 2 & $\mathrm{C}_{15} \mathrm{H}_{26} \mathrm{O}$ & 10 \\
\hline 12.525 & Caryophyllene oxide & $\mathrm{C}_{15} \mathrm{H}_{24} \mathrm{O}$ & 10 \\
\hline 15.152 & 2-Azidomethyl-1,3,3-trimethyl-cyclohexene & $\mathrm{C}_{10} \mathrm{H}_{17} \mathrm{~N}_{3}$ & 10 \\
\hline 15.423 & Hexanoic acid, 3,7-dimethyl-2,6-octadienyl ester,(E)- & $\mathrm{C}_{16} \mathrm{H}_{28} \mathrm{O}_{2}$ & 10 \\
\hline 17.258 & Farnesol, acetate & $\mathrm{C}_{16} \mathrm{H}_{28} \mathrm{O}_{2}$ & 10 \\
\hline 20.158 & 2,6-Octadien-1-ol, 3,7-dimethyl-, propanoate, (Z)- & $\mathrm{C}_{13} \mathrm{H}_{22} \mathrm{O}_{2}$ & 10 \\
\hline
\end{tabular}


Table 2: IUPAC Name, 2D, 3D structure of bioactive compounds in CMEO

\begin{tabular}{|c|c|c|}
\hline IUPAC Name & $\begin{array}{c}\text { 2D } \\
\text { Chemical Structure }\end{array}$ & $\begin{array}{c}\text { 3D } \\
\text { Chemical Structure }\end{array}$ \\
\hline \multicolumn{3}{|l|}{ Cyclodecyne; 4-Decen-6-yne, (Z) } \\
\hline \multicolumn{3}{|c|}{ 2-Ethylimino-4-methyl-pent-3-enenitrile } \\
\hline \multicolumn{3}{|l|}{ Dihydrocarvyl acetate } \\
\hline \multicolumn{3}{|l|}{ 2-Methylbenzaldehyde } \\
\hline \multicolumn{3}{|l|}{ Geranyl butyrate } \\
\hline \multicolumn{3}{|c|}{ 1,5,9,9-Tetramethyl-1,4,7-cycloundecatriene } \\
\hline Caryophyllene oxide & & \\
\hline
\end{tabular}

Table 3: Molecular properties of bioactive compounds in CMEO

\begin{tabular}{|l|l|l|l|l|l|l|l|}
\hline PROPERTY & \multicolumn{7}{|c|}{ BIOACTIVE COMPOUNDS } \\
\hline CID & 137799 & 68315 & 73918 & 998 & 5282854 & 5281522 & 1742210 \\
\hline MF & $\mathrm{C}_{10} \mathrm{H}_{16}$ & $\mathrm{C}_{8} \mathrm{H}_{12} \mathrm{~N}_{2}$ & $\mathrm{C}_{10} \mathrm{H}_{18} \mathrm{O}$ & $\mathrm{C}_{8} \mathrm{H}_{8} \mathrm{O}$ & $\mathrm{C}_{14} \mathrm{H}_{24} \mathrm{O}_{2}$ & $\mathrm{C}_{15} \mathrm{H}_{24}$ & $\mathrm{C}_{15} \mathrm{H}_{24} \mathrm{O}$ \\
\hline miLogP & 4.54 & 2.09 & 3.35 & 2.13 & 4.83 & 5.07 & 4.14 \\
\hline TPSA & 0.00 & 36.16 & 26.30 & 17.07 & 26.30 & 0.00 & 12.53 \\
\hline N atoms & 10 & 10 & 4 & 9 & 16 & 15 & 16 \\
\hline MW (g/mol) & 136.24 & 136.20 & 154.24 & 120.15 & 224.34 & 204.36 & 220.36 \\
\hline Non & 0 & 2 & 2 & 1 & 2 & 0 & 1 \\
\hline n OHNH & 0 & 0 & 0 & 0 & 0 & 0 & 0 \\
\hline N violations & 0 & 0 & 0 & 0 & 0 & 1 & 0 \\
\hline N rotb & 3 & 2 & 3 & 1 & 8 & 234.00 & 234.01 \\
\hline volume & 162.53 & 146.66 & 208.06 & 119.59 & 245.69 & 0 \\
\hline
\end{tabular}


Table 4: Biological properties of compounds in CMEO

\begin{tabular}{|l|l|l|l|l|l|l|l|}
\hline PROPERTIES & \multicolumn{7}{|c|}{ BIOACTIVE COMPOUNDS } \\
\hline CID & $\mathbf{1 3 7 7 9 9}$ & $\mathbf{6 8 3 1 5}$ & $\mathbf{7 3 9 1 8}$ & $\mathbf{9 9 8}$ & $\mathbf{5 2 8 2 8 5 4}$ & $\mathbf{5 2 8 1 5 2 2}$ & $\mathbf{1 7 4 2 2 1 0}$ \\
\hline GPCR ligand & -0.56 & -1.64 & -0.47 & -2.33 & -0.26 & $\mathbf{0 . 0 3}$ & $\mathbf{0 . 0 8}$ \\
\hline $\begin{array}{l}\text { Ion channel } \\
\text { modulator }\end{array}$ & $\mathbf{0 . 5 7}$ & -1.04 & $\mathbf{0 . 2 3}$ & -1.80 & $\mathbf{0 . 0 5}$ & $\mathbf{0 . 1 3 2}$ & $\mathbf{0 . 1 4}$ \\
\hline $\begin{array}{l}\text { Kinase } \\
\text { inhibitor }\end{array}$ & -1.05 & -2.08 & -1.25 & -2.40 & -0.86 & -0.95 & -0.86 \\
\hline $\begin{array}{l}\text { Nuclear } \\
\text { receptor } \\
\text { ligand }\end{array}$ & -0.18 & -2.06 & -0.17 & -2.20 & $\mathbf{0 . 0 3}$ & $\mathbf{0 . 4 0}$ & $\mathbf{0 . 6 2}$ \\
\hline $\begin{array}{l}\text { Protease } \\
\text { inhibitor }\end{array}$ & -0.76 & -1.92 & -0.44 & -2.91 & -0.56 & -0.63 & 0.00 \\
\hline $\begin{array}{l}\text { Enzyme } \\
\text { inhibitor }\end{array}$ & $\mathbf{0 . 4 3}$ & -0.84 & -0.12 & -1.91 & $\mathbf{0 . 3 0}$ & $\mathbf{0 . 4 1}$ & $\mathbf{0 . 5 7}$ \\
\hline
\end{tabular}

Table 5: Summary of MTIR/ DL/DS score of bioactive compounds in CMEO

\begin{tabular}{|l|l|l|l|l|l|l|}
\hline COMPOUND & MP & TP & IP & RE & DL & DS \\
\hline Cyclodecyne; 4-Decen-6-yne, (Z)- & None & None & High & None & -10.80 & 0.21 \\
\hline 2-Ethylimino-4-methyl-pent-3-enenitrile & None & None & None & None & -4.87 & 0.48 \\
\hline Dihydrocarvyl acetate & None & None & High & None & -19.56 & 0.26 \\
\hline 2-Methylbenzaldehyde & None & None & Medium & High & -5.59 & 0.23 \\
\hline Geranyl butyrate & None & None & High & None & -5.84 & 0.21 \\
\hline 1,5,9,9-Tetramethyl-1,4,7-cycloundecatriene & None & None & None & None & -5.08 & 0.28 \\
\hline Caryophyllene oxide & None & Medium & None & Medium & -4.77 & 0.25 \\
\hline
\end{tabular}

Note: $M P$ = Mutagenic property; TP = Toxicology property; IP = Irritant property; RE = Reproductive property; DL = Drug Likeness; DS = Druggable Score

Table 6: Druggability Properties of bioactive compounds in CMEO

\begin{tabular}{|l|l|l|l|l|l|l|}
\hline \multirow{2}{*}{ Druggability Property } & \multicolumn{5}{|c|}{ BIOACTIVE COMPOUNDS } \\
\cline { 2 - 7 } & $\mathrm{C}_{10} \mathrm{H}_{16}$ & $\mathrm{C}_{8} \mathrm{H}_{12} \mathrm{~N}_{2}$ & $\mathrm{C}_{10} \mathrm{H}_{18} \mathbf{O}$ & $\mathrm{C}_{8} \mathrm{H}_{8} \mathbf{O}$ & $\mathrm{C}_{\mathbf{1 4}} \mathrm{H}_{24} \mathbf{O}_{2}$ & $\mathrm{C}_{15} \mathrm{H}_{24}$ \\
\hline Lipinski's rule of 5 violations & 0 & 0 & 0 & 0 & 0 & 0 \\
\hline Veber rule & Good & Good & Good & Good & Good & Good \\
\hline Egan rule & Good & Good & Good & Good & Good & Good \\
\hline Oral PhysChem score & 0 & 1 & 2 & 2 & 1 & 2 \\
\hline GSK's 4/400 score & Good & Good & Good & Good & Good & Good \\
\hline Pfizer's 3/75 score & Garning & Bad & Bad & Bad & Bad & Bad \\
\hline QEDw score & 0.521 & 0.506 & 0.493 & 0.434 & 0.433 & 0.434 \\
\hline Solubility & 12379.28 & 8150.46 & 4750.64 & 5166.30 & 4350.64 & 5166.30 \\
\hline Solubility Index & Good & Good & Good & Good & Good & Good \\
\hline
\end{tabular}

Druggability scoring schemes were computed using FAF-Drugs 4(28961788) and FAF-QED (28961788) open-source Chem-informatics platform.

Studies have led to the isolation of alkaloids, volatile and non-volatile terpenoids, flavonoids, carotenoids and tannins from Cymbopogon species. $\beta$-Caryophyllene from CMEO has been reported to be directly beneficial for colitis, osteoarthritis, diabetes, cerebral ischemia, anxiety and depression, liver fibrosis. Biological activities of these secondary metabolites of Cymbopogon martini (Palmarosa) have been reported for its antitumor, antioxidant, antiinfectious, anti-inflammatory, and analgesic activities and effects on the central nervous system, endocrine system, disorders such as cardiac remodeling after myocardial infarction, body weight changes, dyslipidemia, cerebral ischemia, hepato-nephrotoxicity, stress, and anxiety. Antiinflammatory activity of CMEO has been attributed to the 
presence and synergistic activity of carnosol and carnosic, rosmarinic, ursolic, oleanolic, and micromeric acids (A). Specifically, anti-inflammatory activity has been attributed to synergic effects of ursolic and micromeric acids present in CMEO. These natural drugs can be proposed for preclinical and clinical studies in different diseases and pathological conditions.

\section{CONCLUSION}

Cymbopogon species have been used as traditional medicine in many countries since antiquity. CMEO has been used in traditional and in conventional medicine due to the pharmacological potential of their phytochemicals. C. martini (Palmarosa) contains a large variety of bioactive molecules with great therapeutic potential and biological activities such as insecticidal, anti-protozoan, anticancer, anti-HIV, antiinflammatory and anti-diabetes effects. CMEO has remarkable anti-inflammatory, antimicrobial, and antioxidant properties, which have been extensively reported in several formulations. However, development of new formulations containing other less common CMEO extracts is warranted through trials to establish the credentials of pharmacologically active phyto-compounds towards safety/ efficacy, in treating various pathological conditions including COVID-19 and other viral infections owing to the physiochemical properties and druggable nature of CMEO.

\section{REFERENCES}

1. Jummes B, Sganzerla WG, da Rosa CG, Noronha CM, Nunes MR, Bertoldi FC, Barreto PL. Antioxidant and antimicrobial poly- $\varepsilon$ caprolactone nanoparticles loaded with Cymbopogon martinii essential oil. Biocatalysis and Agricultural Biotechnology. 2020; 23:101499. https://doi.org/10.1016/j.bcab.2020.101499

2. Avoseh O, Oyedeji O, Rungqu P, Nkeh-Chungag B, Oyedeji A. Cymbopogon species; ethnopharmacology, phytochemistry and the pharmacological importance. Molecules. 2015; 20(5):743853. https://doi.org/10.3390/molecules 20057438

3. Prashar A, Hili P, Veness RG, Evans CS. Antimicrobial action of palmarosa oil (Cymbopogon martinii) on Saccharomyces cerevisiae. Phytochemistry. 2003; 63(5):569-75. https://doi.org/10.1016/S0031-9422(03)00226-7

4. Raina VK, Srivastava SK, Aggarwal KK, Syamasundar KV, Khanuja SP. Essential oil composition of Cymbopogon martinii from different places in India. Flavour and Fragrance Journal. 2003; 18(4):312-5. https://doi.org/10.1002/ffj.1222

5. Kumar R, Srivastava M, Dubey NK. Evaluation of Cymbopogon martinii oil extract for control of postharvest insect deterioration in cereals and legumes. Journal of food protection. 2007; 70(1):172-8. https://doi.org/10.4315/0362-028X70.1 .172

6. Kalagatur NK, Nirmal Ghosh OS, Sundararaj N, Mudili V. Antifungal activity of chitosan nanoparticles encapsulated with Cymbopogon martinii essential oil on plant pathogenic fungi Fusarium graminearum. Frontiers in pharmacology. 2018; 9:610. https://doi.org/10.3389/fphar.2018.00610

7. Mishra PK, Kedia A, Dubey NK. Chemically characterized Cymbopogon martinii (Roxb.) Wats. essential oil for shelf life enhancer of herbal raw materials based on antifungal, antiaflatoxigenic, antioxidant activity and favorable safety profile. Plant Biosyst. 2015; 150:1313-1322

https://doi.org/10.1080/11263504.2015.1054450

8. Caballero-Gallardo K, Olivero-Verbel J, Stashenko EE. Repellency and toxicity of essential oils from Cymbopogon martinii, Cymbopogon flexuosus and Lippia origanoides cultivated in Colombia against Tribolium castaneum. Journal of Stored Products Research. 2012; 50:62-5. https://doi.org/10.1016/j.jspr.2012.05.002

9. Andrade BF, Conti BJ, Santiago KB, Fernandes A, Sforcin JM. C ymbopogon martinii essential oil and geraniol at non-cytotoxic concentrations exerted immunomodulatory/anti-inflammatory effects in human monocytes. Journal of Pharmacy and Pharmacology. 2014; 66(10):1491-6. https://doi.org/10.1111/jphp.12278

10. Al-Shalah LA, Hindi NK, Mohsen IH. Essential Oils. Essential Oils: Bioactive Compounds, New Perspectives and Applications. 2020; 9:29.

11. Chávez-González ML, Rodríguez-Herrera R, Aguilar CN. Essential oils: A natural alternative to combat antibiotics resistance. Antibiotic Resistance-Mechanisms and New Antimicrobial Approaches; Kon, K., Rai, M., Eds. 2016; 227-37. https://doi.org/10.1016/B978-0-12-803642-6.00011-3

12. Atanasov AG, Waltenberger B, Pferschy-Wenzig EM, Linder T, Wawrosch C, Uhrin P, Temml V, Wang L, Schwaiger S, Heiss EH, Rollinger JM. Discovery and resupply of pharmacologically active plant-derived natural products: A review. Biotechnology advances. 2015; 33(8):1582-614. https://doi.org/10.1016/j.biotechadv.2015.08.001

13. Rašković A, Milanović I, Pavlović N, Ćebović T, Vukmirović S, Mikov M. Antioxidant activity of rosemary (Rosmarinus officinalis L.) essential oil and its hepatoprotective potential. BMC complementary and alternative medicine. 2014; 14(1):1-9. https://doi.org/10.1186/1472-6882-14-225

14. Khan MS, Ahmad I. In vitro antifungal, anti-elastase and antikeratinase activity of essential oils of Cinnamomum-, Syzygiumand Cymbopogon-species against Aspergillus fumigates and Trichophyto rubrum. Phytomedicine. 2011; 19:48-55 https://doi.org/10.1016/j.phymed.2011.07.005

15. Yoon MY, Cha B, Kim JC Recent trends in studies on botanical fungicides in agriculture. The plant pathology journal. 2013; 29(1):1. https://doi.org/10.5423/PPJ.RW.05.2012.0072

16. Matthew KM. Flora of the Tamilnadu Carnatic. The Rapinat Herbarium, St. Joseph's College, Tiruchirapalli, India; 1981

17. Gamble JS, Fischer CE. Flora of the Presidency of Madras. London, UK: West, Newman and Adlard; 1915. https://doi.org/10.5962/bhl.title.21628

18. Eleyinmi AF Chemical composition and antibacterial of Gongronema latifolium J Zhejiang Univ Sci B 2007; 8:352-358 https://doi.org/10.1631/jzus.2007.B0352

19. Zhou Y, Wu F, Li L, Shen X, Chen G, Wang X, Liang X, Tan M, Huang Z. Computational approaches in preclinical studies on drug discovery and development. Frontiers in Chemistry. 2020 8:726-31. https://doi.org/10.3389/fchem.2020.00726

20. Ramya S, Neethirajan K, Jayakumararaj R. Profile of bioactive compounds in Syzygium cumini-a review. Journal of Pharmacy research. 2012; 5(8):4548-53.

21. Gaba J, Bhardwaj G, Sharma A. Lemongrass. In Antioxidants in Vegetables and Nuts-Properties and Health Benefits 2020 (pp. 75-103). Springer, Singapore. https://doi.org/10.1007/978981-15-7470-2_4 